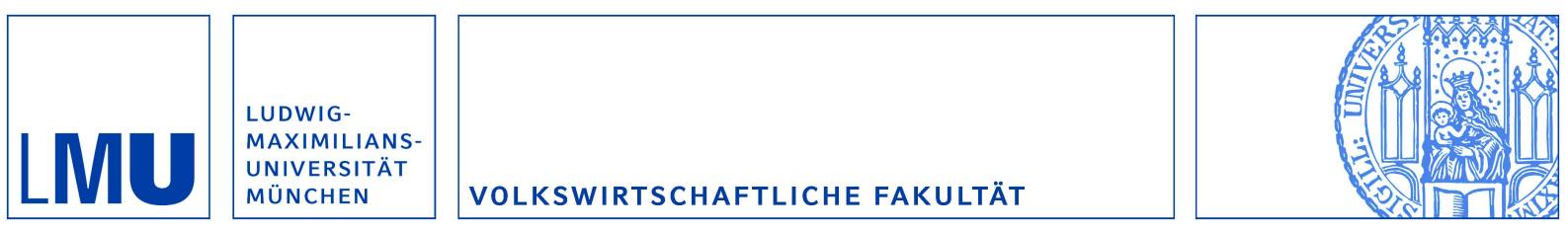

Herweg, Fabian:

The Expectation-Based Loss-Averse Newsvendor

Munich Discussion Paper No. 2012-23

Department of Economics

University of Munich

Volkswirtschaftliche Fakultät

Ludwig-Maximilians-Universität München

Online at https://doi.org/10.5282/ubm/epub. 14065 


\title{
THE EXPECTATION-BASED LOSS-AVERSE NEWSVENDOR
}

\author{
FABIAN HERWEG
}

\begin{abstract}
We modify the classic single-period inventory management problem by assuming that the newsvendor is expectation-based loss averse according to Köszegi and Rabin (2006, 2007). Expectation-based loss aversion leads to an endogenous psychological cost of leftovers as well as stockouts. If there are no monetary stockout costs, then the loss-averse newsvendor orders a quantity lower than the quantity ordered by a profit-maximizing newsvendor. If there are positive monetary costs associated with stockouts, then the loss-averse newsvendor places suboptimal orders, which can be either too high or too low.
\end{abstract}

Keywords: behavioral operations management; inventory decision; loss aversion; newsvendor

\section{INTRODUCTION}

A standard model in stochastic inventory management is the single-period newsvendor problem, which is widely used and analyzed in operations management since Arrow, Harris, and Marschak (1951). The eponymous story is the following: A newsgirl must decide how many newspapers to order in the morning from the publisher for sale during the day. The newsgirl sells the newspapers at a fixed mark-up per unit. Her problem is that she does not know how many customers will stop by at her newsstand during the day when placing the order. If she orders too many newspapers, she must salvage all unsold newspapers to the publisher at a low value. If she orders too few, she will have missed an opportunity for additional sales and might also face additional shortage costs such as a loss of goodwill. Thus, the newsgirl faces a trade-off in balancing her loss in profits due to ordering too many (leftovers) against her loss in profits of ordering too few newspapers (stockouts). The newsvendor model is reflective of many real life situations with analogies in topics such as capacity planning, yield management, and supply chain contracts.

In the last two decades, empirical investigations using field data as well as laboratory experiments have shown that actual orders often deviate from the standard prediction based on a risk-neutral profit-maximizing newsgirl. ${ }^{1}$ For instance, analyzing the inventory management decisions of the skiwear firm Sport Obermeyer, Fisher and Raman (1996) concluded that the managers consistently ordered too little. Experimental evidence, like Schweitzer and Cachon (2000), document that average orders are typically biased in the direction of the median demand realization, a phenomenon called pull-to-center bias. Even though orders are distorted towards

Date: October 1, 2012.

The author would like to thank René Cyranek, Heiko Karle, Jo Maier, Daniel Müller, Marta Serra-Garcia, Klaus M. Schmidt, and Philipp B. Weinschenk for their feedback. Financial support by the Deutsche Forschungsgemeinschaft through SFB-TR 15 is gratefully acknowledged.

${ }^{1}$ The fact that actual order quantities deviate from the profit-maximizing quantity is shown for field data by Fisher and Raman (1996) and Katok, Lathrop, Tarantino, and Xu (2001) and for experimental subjects by Schweitzer and Cachon (2000) and Bostian, Holt, and Smith (2008). 
the median, Becker-Peth, Katok, and Thoenemann (2011) find that experimental subjects have a tendency to order less than the profit-maximizing quantity.

Several papers have tried to explain the departure of actual order quantities from the profit-maximizing order. With the newsgirl making a decision under uncertainty her behavior might be driven by risk preferences (Eeckhouldt, Gollier, and Schlesinger, 1995). Modeling the newsgirl's preferences based on expected utility theory (EUT), however, often leads to implausible comparative statics, e.g., the order quantity decreases if the retail price increases (Wang, Webster, and Suresh, 2009).

Due to the recent developments in behavioral operations management, nonstandard risk preferences have been applied to the newsvendor problem. Already MacCrimmon and Wehrung (1988) documented, based on questionnaire responses, that managerial decision making under uncertainty is consistent with loss aversion (Kahneman and Tversky, 1979). The loss-averse newsgirl compares her actual profit to a reference profit and feels a loss if actual profits are lower than the reference level. A crucial question regarding loss aversion is the following: What shapes the reference point? Existing theories of a loss-averse newsgirl assume a fixed and exogenously given reference point (Wang and Webster, 2009; Schweitzer and Cachon, 2000). Under the ad hoc assumption of an exogenous reference point, the newsgirl's order quantity crucially depends on the selected reference point. In contrast to the existing literature, we posit that the newsgirl is expectation-based loss averse à la Köszegi and Rabin $(2006,2007)$. With the newsgirl having experience - or at least access to historical data - it seems reasonable to assume that her reference point is (at least partly) shaped by expectations. Support for the hypothesis that the reference point is determined by lagged expectations is provided by Abeler, Falk, Goette, and Huffman (2011) and Ericson and Fuster (2011). ${ }^{2}$

According to the concept of Köszegi and Rabin, the newsgirl derives utility from the monetary outcome as well as from comparing her actual profits with her lagged expectations. Crucially, the reference point is fully determined by the newsgirl's rational expectations. Thus, the reference point is endogenously determined, i.e., there is no degree of freedom for the modeler in selecting the reference point. In our model this implies that, given her actual order quantity, the newsgirl feels a loss compared to all possible demand states that would have resulted in higher profits. With the newsgirl's profit being maximized if actual demand coincides with the ordered quantity, expectation-based loss aversion introduces an endogenous psychological cost of stockouts as well as of leftovers into the newsvendor model. The relative magnitude of the aversion towards stockouts compared to the aversion of leftovers depends on the monetary losses associated with these two types of ex-post inventory errors.

If there is almost no shortage penalty, the newsgirl's aversion towards stockouts is low and she orders a quantity which is lower than the profit-maximizing quantity in order to reduce her expected psychological costs of leftovers (Theorem 2). This result is also obtained for a risk-averse newsgirl within the expected utility framework. Moreover, the optimal order of the loss-averse newsgirl displays plausible comparative statics with respect to all price and cost parameters, which is in contrast to findings obtained for a risk-averse newsgirl. The analysis becomes more intricate

\footnotetext{
${ }^{2}$ They do not consider a newsvendor setting in order to show that expectations shape a reference point.
} 
in the case where there is a monetary cost associated with stockouts. In this case, the newsgirl balances, with the ordering decision, her expected disappointment due to stockouts against her expected disappointment due to leftovers. Nevertheless, for many numerical examples, the loss-averse newsgirl orders less than the profitmaximizing quantity, e.g., always if demand is uniformly distributed (Proposition 2). We show, however, that the order placed by a loss-averse newsgirl can be higher as well as lower than the profit-maximizing quantity (Example 3). Despite the analytical complexity in characterizing the optimal order placed by a loss-averse newsgirl for general demand distributions, we can show that there is a unique optimal order quantity (Theorem 3) and that the difference between the placed order and the profit-maximizing order increases in the degree of loss aversion (Theorem 4). Put differently, the direction of the distortion in the order quantity is independent of the degree of loss aversion.

Loss aversion is not the only type of reference-dependent preferences which is discussed in the economic as well as psychological literature. Another important type of reference-dependent preferences is regret aversion (Bell, 1982). Here, the newsgirl is not disappointed about the realized demand, but regrets that she has not ordered a quantity closer to actual demand. In her ex-ante ordering decision, the regret averse newsgirl takes her anticipated regret into account. We show that regret aversion cannot explain departures from profit-maximizing orders (Proposition 3).

The paper is organized as follows. After briefly reviewing the related literature, we introduce the newsvendor model in Section 2. The optimal order quantity of a lossaverse newsvendor for the cases without shortage penalty and with shortage penalty are analyzed in Section 3 and Section 4, respectively. In Section 5 we consider a newsvendor who is regret averse. Finally, in Section 6 we outline our conclusions and summarize the main findings.

Literature. The first analysis of the newsvendor model is due to Edgeworth (1888), who introduced it to study cash holdings of banks. ${ }^{3}$ Arrow, Harris, and Marschak (1951) derive the by now well-known critical fractile solution for a profit-maximizing newsvendor. A risk-averse newsvendor is analyzed by Eeckhouldt, Gollier, and Schlesinger (1995). They show that comparative statics with respect to prices and costs are often ambiguous and can have unintuitive signs, e.g., the optimal order can be increasing in the wholesale price and decreasing in the retail price. Intuitive comparative statics can be obtained when assuming decreasing partial relative risk aversion. Wang, Webster, and Suresh (2009) not only show that the order placed by a risk-averse newsvendor often decreases in the retail price but also that it can become arbitrarily small if the retail price is sufficiently high.

More recent contributions to the behavioral operations management literature incorporate preferences which are beyond the EUT-framework (Wang and Webster, 2009; Ho, Lim, and Cui, 2010; Croson, Croson, and Ren, 2008). The main purpose of these articles is to explain the so-called "pull-to-center" bias, which has been documented by experimental data - most prominently by Schweitzer and Cachon (2000). Schweitzer and Cachon (2000) also discuss several potential theoretical explanations for the pull-to-center bias, including a simple model of loss aversion. They argue

\footnotetext{
${ }^{3}$ For excellent reviews about the newsvendor model see Moutaz and Khouja (1999) and Qin, Wang, Vakharia, Chen, and Seref (2011).
} 
that the only models consistent with their findings are (i) a preference for minimizing ex-post inventory error, which is a kind of regret aversion, and (ii) an anchoring and insufficient adjustment bias. The preferences for minimizing ex-post inventory error are refined by Ho, Lim, and Cui (2010) in order to explain even more stylized facts regarding the pull-to-center bias. They also conduct an experiment of their multilocation newsvendor model which confirms the pull-to-center bias. A different explanation for the pull-to-center bias is provided by Croson, Croson, and Ren (2008), who argue that the newsvendor might suffer from the overprecision bias, i.e., she underestimates the variance of the demand distribution. ${ }^{4}$ The existence of the pull-to-center bias has recently been questioned by Lau, Bearden, and Hasija (2012). They argue that the pull-to-center bias might be an artifact of using aggregate (averaged) data and that this bias does not exist for individual ordering decisions.

The contribution closest related to our paper is Wang and Webster (2009). They extend the loss-aversion model developed by Schweitzer and Cachon (2000) by allowing for a shortage cost. They show that a loss-averse newsvendor orders less than the profit-maximizing quantity if the shortage cost is low and more if the shortage cost is high. Moreover, the optimal order quantity may increase in the wholesale price and decrease in the retail price. While Wang and Webster (2009) focus on a fixed exogenous reference point, we consider a reference point which is endogenously determined by the newsvendor's rational expectations. Moreover, given shortage costs are low, we derive reasonable comparative statics for all price and cost parameters.

\section{The Newsvendor Model}

We consider a three-node supply chain consisting of a supplier, a newsvendor, and customers. The newsvendor sells a short-life-cycle product with stochastic demand. At the beginning of the selling period the newsvendor chooses an optimal stocking policy without knowing total customer demand. The newsvendor orders quantity $q$ at a fixed wholesale price $w$ per unit. During the selling season replenishments are not possible. The newsvendor faces demand $D(\cdot)=x$ at the fixed retail price $p$. Demand is a non-negative random variable, which is distributed according to the twice continuously differentiable cumulative distribution function $F(x)$ over the interval $I=[\underline{x}, \bar{x}]$, with $0 \leq \underline{x}<\bar{x}$. Let $f(x)>0$ be the corresponding probability density function. The supplier operates without capacity constraints and zero lead time of supply. Thus, the order placed by the newsvendor with the supplier at the beginning of the selling season will immediately be filled. Sales of the product occur during the selling season. If realized demand is lower than the stocked quantity $(x<q)$, then $q-x$ units of the product are left over at the end of the selling period. These $q-x$ units are salvaged at a unit value $s$ by the newsvendor. If, on the other hand, realized total customer demand is higher than the stocked quantity $(x>q)$, then there is an unsatisfied excess demand of $x-q$ units. In this case a shortage cost penalty $c \geq 0$ is incurred per unit of excess demand. The shortage cost can account for the costs of emergency deliveries and fair dealing costs that might arise for the newsvendor in order to reduce the negative effects of stockouts on future

\footnotetext{
${ }^{4} \mathrm{~A}$ model of bounded rationality which can generate predictions consistent with experimental data is presented by $\mathrm{Su}(2008)$. He assumes that the newsvendor has standard preferences but makes decisions with noise.
} 
sales. Further, we assume that the newsvendor has an unbiased forecast of the total demand and knows $F(x) .{ }^{5}$

Assumption 1. It holds that $0 \leq s<w<p$.

Assumption 1 implies (i) that there is a positive mark-up on the product, $p-w>0$, and (ii) that salvaging units is costly for the newsvendor, $s-w<0$.

2.1. The Profit-Maximizing Newsvendor. The newsvendor's profit at the end of the selling season is

$$
\pi(q, x)= \begin{cases}\hat{\pi}(q, x) \equiv(p-w) x-(w-s)(q-x) & \text { if } x<q \\ \tilde{\pi}(q, x) \equiv(p-w) q-c(x-q) & \text { if } x \geq q\end{cases}
$$

The newsvendor cannot observe actual profits at the beginning of the selling period when placing her order with the supplier because customer demand has not been realized. The traditional approach to analyze the problem is to assume that the newsvendor is a risk and loss neutral rational decision maker who maximizes expected profits. Ex ante the newsvendor expects that realized demand will be lower than the ordered quantity with probability $F(q)$ and higher with probability $1-F(q)$. Thus, the newsvendor's expected profit at the beginning of the selling season is:

$$
\begin{aligned}
E \pi(q)=-(w-s) q F(q)+(p-s) & \int_{\underline{x}}^{q} x f(x) d x \\
& +(p+c-w) q[1-F(q)]-c \int_{q}^{\bar{x}} x f(x) d x .
\end{aligned}
$$

It is readily verified that the expected profit (2) is strictly concave in $q$ and maximized at $q^{*}$ implicitly characterized by

$$
F\left(q^{*}\right)=\frac{p+c-w}{p+c-s} \in(0,1) .
$$

This is the well-known critical fractile solution for the single-period newsvendor problem (Arrow, Harris, and Marschak, 1951). The ratio $(p+c-w) /(p+c-s)$ is called the critical fractile and sometimes used to classify products as either highprofit or low-profit products, depending on whether the critical fractile is larger or smaller than one-half.

The following comparative statics regarding the optimal order quantity are immediately at hand:

$$
\frac{\partial q^{*}}{\partial p}>0, \quad \frac{\partial q^{*}}{\partial c}>0, \quad \frac{\partial q^{*}}{\partial w}<0, \quad \text { and } \quad \frac{\partial q^{*}}{\partial s}>0 .
$$

2.2. The Loss-Averse Newsvendor. We posit that the newsvendor is expectationbased loss averse in the sense of Köszegi and Rabin (2006, 2007). By this concept, overall utility of the newsvendor is assumed to have two components: intrinsic utility and gain-loss utility. Intrinsic utility equals the realized profit because we abstract from standard risk aversion. Gain-loss utility is derived by comparing the actual profit to a reference level $r$. Thus, the newsvendor's overall utility ex post can be written as

$$
U=\pi(q, x)+m(r-\pi(q, x)),
$$

\footnotetext{
${ }^{5}$ Except for being loss averse the newsvendor is fully rational.
} 
where $m(\cdot)$ is the gain-loss function. For simplicity we abstract from diminishing sensitivity and assume a piece-wise linear gain-loss function:

$$
m(z)=-\lambda[z]^{+},
$$

with $[z]^{+}=z$ for $z>0$ and $[z]^{+}=0$ for $z \leq 0$. Moreover, $\lambda \geq 0$, i.e., the newsvendor is loss averse for $\lambda>0$ and for $\lambda=0$ we are back in the standard case of a profit-maximizing newsvendor. ${ }^{6}$

Following Köszegi and Rabin $(2006,2007)$, the newsvendor's reference point is determined by her rational expectations about demand and thus profits. A given profit is then evaluated by comparing it to all possible profits for the given order quantity, where each comparison is weighted with the ex-ante probability with which the alternative profit occurs. With the actual profit being itself uncertain, the newsvendor's expected utility is obtained by averaging over all these comparisons. We apply the concept of choice-acclimating personal equilibrium (CPE) as defined in Köszegi and Rabin (2007), which assumes that a decision maker correctly predicts her choice set, the environment she faces, in particular the set of possible outcomes and how the distribution of these outcomes depends on her decisions. The eponymous feature of CPE is that the newsvendor's reference point is affected by her choice of action, here the ordered quantity. As pointed out by Köszegi and Rabin, CPE refers to the analysis of risk preferences regarding outcomes that are resolved long after all decisions are made. Hence, this concept seems well-suited for the newsvendor problem because the newsvendor places her order before the demand uncertainty is resolved. The newsvendor's utility ex post, from order quantity $q$ if demand $x$ is realized, is given by

$$
U(q, x)=\pi(q, x)-\lambda \int_{\underline{x}}^{\bar{x}}[\pi(q, z)-\pi(q, x)]^{+} f(z) d z .
$$

The newsvendor's expected utility is not necessarily concave due to the strong notion of risk aversion implied by the CPE concept. The notion of risk aversion is strong in the sense that the decision maker may prefer stochastically dominated options if $\lambda>1$ (Köszegi and Rabin, 2007). The reason is that for $\lambda>1$ the decision maker's primary concern ex ante is to reduce the scope of possibly feeling a loss ex post. The decision maker would rather give up the hope of a very favorable outcome in order to avoid the disappointment in case of not obtaining the favorable outcome. In order to guarantee that the newsvendor's problem is concave, we assume that the weight attached to loss utility does not exceed the weight attached to intrinsic utility. ${ }^{7}$

Assumption 2. No dominance of loss utility, $\lambda \leq 1$.

The expectation-based loss-averse newsvendor places an order with the supplier $q_{\lambda}$ that maximizes her expected utility at the beginning of the selling season. Thus, $q_{\lambda}$ maximizes

$$
E U(q)=\int_{\underline{x}}^{\bar{x}} \pi(q, x) f(x) d x-\lambda \int_{\underline{x}}^{\bar{x}} \int_{\underline{x}}^{\bar{x}}[\pi(q, z)-\pi(q, x)]^{+} f(z) f(x) d z d x .
$$

\footnotetext{
${ }^{6}$ Given the gain-loss function is piece-wise linear, we can focus on loss utility without loosing generality. Gain utility does not affect the newsvendor's decision because for her ex ante order only expected net losses matter.

${ }^{7}$ Assumption 2 is not uncommon in the literature and also imposed, for instance, by Herweg, Müller, and Weinschenk (2010) and Herweg and Mierendorff (forthcoming).
} 
We start the analysis of the loss-averse newsvendor with a simpler version of the stochastic inventory problem. In the following section, we assume that there is no shortage cost, i.e., $c=0$. As we will explain below, without a shortage cost, for a given demand realization $x$ the newsvendor feels a loss compared to all possible higher demand realizations. This case allows us to obtain first insights into the behavior of a loss-averse newsvendor.

\section{The Newsvendor Problem without Shortage Cost}

In this section, we assume that if realized demand is higher than the stocked quantity, then the newsvendor sells $q$ units at price $p$ as before but does not incur a shortage penalty on $x-q$ units, i.e., $c=0 .{ }^{8}$ The newsvendor's profit at the end of the selling season simplifies to:

$$
\pi(q, x)= \begin{cases}\hat{\pi}(q, x) \equiv(p-w) x-(w-s)(q-x) & \text { if } x<q \\ \tilde{\pi}(q, x) \equiv(p-w) q & \text { if } x \geq q\end{cases}
$$

Without shortage costs, the optimal order quantity of a profit-maximizing newsvendor $q^{*}$ is characterized by $F\left(q^{*}\right)=(p-w) /(p-s)$.

It is important to note that the newsvendor's ex-post profit is strictly increasing in the actual demand for $x<q$ and independent of the actual demand for $x \geq q$. For a low actual demand realization, $x<q$, the newsvendor feels a loss compared to all possible demand realizations $z>x$. Thus, for $x<q$ the ex-post utility of the loss-averse newsvendor is given by,

$$
\hat{U}(q, x)=\hat{\pi}(q, x)-\lambda \int_{x}^{q}[\hat{\pi}(q, z)-\hat{\pi}(q, x)] f(z) d z-\lambda \int_{q}^{\bar{x}}[\tilde{\pi}(q, z)-\hat{\pi}(q, x)] f(z) d z .
$$

Using (6), the above utility can be written as

(7) $\hat{U}(q, x)=(p-s) x-(w-s) q-\lambda(p-s) \int_{x}^{q}[z-x] f(z) d z-\lambda(p-s)(q-x)[1-F(q)]$.

If the realized demand is relatively high, $x \geq q$, then the newsvendor achieves the highest possible profit and thus does not feel any sensations of a loss. This is due to the fact that there is no shortage penalty in the case of excess demand. Thus, for $x \geq q$, the ex-post utility is

$$
\tilde{U}(q, x)=(p-w) q .
$$

At the beginning of the selling season - when the newsvendor places her order-her expected utility as a function of the order quantity is

$$
\begin{aligned}
& E U(q)=\int_{\underline{x}}^{q}\left\{(p-s) x-(w-s) q-\lambda(p-s) \int_{x}^{q}[z-x] f(z) d z-\right. \\
&\lambda(p-s)(q-x)[1-F(q)]\} f(x) d x+(p-w) q[1-F(q)] d x .
\end{aligned}
$$

The loss-averse newsvendor's expected utility is the expected profit minus the expected loss weighted by $\lambda$. For $\lambda=0$ the newsvendor simply maximizes the expected profit. The loss-averse newsvendor chooses an order quantity that balances the tradeoff between maximizing expected profits and minimizing the expected losses. The

\footnotetext{
${ }^{8}$ Several papers that analyze optimal orders of newsvendors who are not profit maximizers focus on the case without a shortage penalty, e.g., Schweitzer and Cachon (2000); Croson, Croson, and Ren (2008); Ho, Lim, and Cui (2010).
} 
newsvendor incurs a loss ex post if actual demand is lower than the stocked quantity. The newsvendor expects this to happen ex ante with probability $F(q)$. Hence, by choosing a lower quantity - and thus also a lower $F(q)$ - the newsvendor can reduce the scope of feeling a loss ex post.

Theorem 1. Suppose there is no shortage penalty, i.e., $c=0$ and that Assumption 2 holds. Then, $\operatorname{EU}(q)$ is strictly concave for all $q \in[\underline{x}, \bar{x}]$ and there is a unique optimal order quantity $q_{\lambda}$ characterized by the following first-order condition:

$$
(p-w)-(p-s) F\left(q_{\lambda}\right)-\lambda(p-s) F\left(q_{\lambda}\right)\left[1-F\left(q_{\lambda}\right)\right]=0 .
$$

Proof. Taking the first derivative of the expected utility (9) with respect to $q$ yields

$$
E U^{\prime}(q)=(p-w)-(p-s) F\left(q_{\lambda}\right)-\lambda(p-s) F\left(q_{\lambda}\right)\left[1-F\left(q_{\lambda}\right)\right] .
$$

By setting this partial derivative equal to zero we obtain (10). Note that corner solutions can be ruled out, because $\left.E U^{\prime}(q)\right|_{q=\underline{x}}=(p-w)>0$ and $\left.E U^{\prime}(q)\right|_{q=\bar{x}}=$ $-(w-s)<0$. The second derivative of expected utility with respect to $q$ is given by

$$
E U^{\prime \prime}(q)=-(p-s) f(q)[1-\lambda+2 \lambda(1-F(q))],
$$

which is negative for all $q$, because of Assumption 2 .

From the first-order condition (10) it becomes apparent that a crucial determinant of the optimal order quantity for a loss averse newsvendor is the so called loss probability. The newsvendor feels a loss with probability $F(q)$ but not compared to all possible demand states only compared to higher states, which occur with probability $1-F(q)$. Thus, in a sense, the newsvendor expects ex ante to feel a loss ex post with "probability" $F(q)(1-F(q))$.

As already conjectured above, a loss-averse newsvendor orders a quantity that is lower than the profit-maximizing quantity.

Theorem 2. Suppose there is no shortage penalty, i.e., $c=0$ and that Assumption 2 holds. Then, $q_{\lambda}<q^{*}$ and $\partial q_{\lambda} / \partial \lambda<0$.

Proof. We evaluate the first-order condition (10) at the profit-maximizing quantity, $\left.E U^{\prime}(q)\right|_{q=q^{*}}=-\lambda(p-s) F\left(q^{*}\right)\left[1-F\left(q^{*}\right)\right]<0$. The derivative is negative for $\lambda>0$ and because $0<F\left(q^{*}\right)<1$. By Theorem 1 the expected utility is strictly concave, and thus $q_{\lambda}<q^{*}$.

By the implicit function theorem, from (10) we obtain:

$$
\frac{\partial q_{\lambda}}{\partial \lambda}=-\frac{F\left(q_{\lambda}\right)\left[1-F\left(q_{\lambda}\right)\right]}{f\left(q_{\lambda}\right)\left[1+\lambda-2 \lambda F\left(q_{\lambda}\right)\right]}<0,
$$

which concludes the proof.

According to Theorem 2 the loss-averse newsvendor orders a quantity which is too low from the profit-maximizing point of view. Moreover, the downward distortion in the order quantity is increasing in the newsvendor's degree of loss aversion. Expectation-based loss aversion is a kind of risk preferences in the sense that a loss-averse decision maker dislikes variations in payments. Note that for $q=\underline{x}$ the newsvendor's profit is independent of the demand realization, i.e., by choosing a lower quantity than $q^{*}$ the newsvendor reduces the variations in potential profits. 
The behavior of the loss-averse newsvendor is similar to the behavior of the riskaverse newsvendor within the EUT framework. The risk-averse as well as the lossaverse newsvendor orders less than the profit-maximizing quantity. The order placed by a standard risk-averse newsvendor displays ambiguous and often counterintuitive comparative statics. As the next proposition shows, comparative statics of changes of the fixed cost and price parameters on the optimal order of the loss-averse newsvendor, however, are unambiguous with intuitive signs.

Proposition 1. Suppose there is no shortage penalty, i.e., $c=0$ and that Assumption 2 holds. The optimal order of the loss-averse newsvendor has the following properties:

$$
\frac{\partial q_{\lambda}}{\partial p}>0, \quad \frac{\partial q_{\lambda}}{\partial s}>0, \quad \text { and } \quad \frac{\partial q_{\lambda}}{\partial w}<0
$$

Proof. The derivatives can readily be obtained from applying the implicit function theorem on the first-order condition (10).

The comparative statics results are the same as for a profit-maximizing newsvendor and also fairly intuitive. If the retail price increases, then the mark-up increases, and thus it is optimal for the newsvendor to order a higher quantity. If, on the other hand, the wholesale price increases and thus the mark-up decreases, the newsvendor optimally orders less. Finally, if the salvage value of the product increases and thus having an excess stock becomes less costly, the newsvendor places a higher order.

A question that is immediately at hand is whether the supplier can achieve supply chain coordination by adjusting the wholesale or the retail price. ${ }^{9}$ For several reasons it might not be in the interest of the supplier to change the retail price, most importantly because this should have an effect on total customer demand. Therefore, we assume that the supplier chooses a wholesale price $\tilde{w}$ when the effective costs for the supplier are $w$ in order to achieve supply chain coordination. Coordination is achieved if the loss-averse newsvendor places an order equal to the profit-maximizing quantity, i.e., if $q_{\lambda}(\tilde{w})=q^{*}$. It is readily verified that the loss-averse newsvendor orders $q^{*}$ for $\tilde{w}=w-\lambda(w-s)(p-w) /(p-s)$. Thus, the order quantity placed by a loss-averse newsvendor is not distorted if she receives a certain discount from the supplier.

Example 1. Suppose that demand is uniformly distributed on the unit interval, i.e., $x \sim U[0,1]$. Here, the profit-maximizing newsvendor orders $q^{*}=(p-w) /(p-s)$ and the loss-averse newsvendor orders

$$
q_{\lambda}=\frac{1}{2 \lambda}\left(\lambda+1-\sqrt{(1-\lambda)^{2}+4 \lambda \frac{w-s}{p-s}}\right) .
$$

The following figures depict the order quantity of the loss-averse newsvendor relative to the profit-maximizing quantity, $q_{\lambda} / q^{*}$.

The Figures 1-4 show the relative distortion in the stocked quantity in dependence of the degree of loss aversion $\lambda \in[0,1]$. In Figure 1 , where $F\left(q^{*}\right) \approx .5$, the relative distortion is (roughly) linearly increasing in $\lambda$. For high-profit products $\left(F\left(q^{*}\right)>.5\right)$-Figures 2 and 4 - the relative distortion is a concave function, i.e., the relative distortion is low for low and moderate degrees of loss aversion. The quantity distortion is particularly low if the salvage value is high and thus the costs

\footnotetext{
${ }^{9}$ Supply chain coordination is achieved if the joint profits from the supplier and the newsvendor are maximized.
} 
Optimal relative order quantity of the loss-averse newsvendor

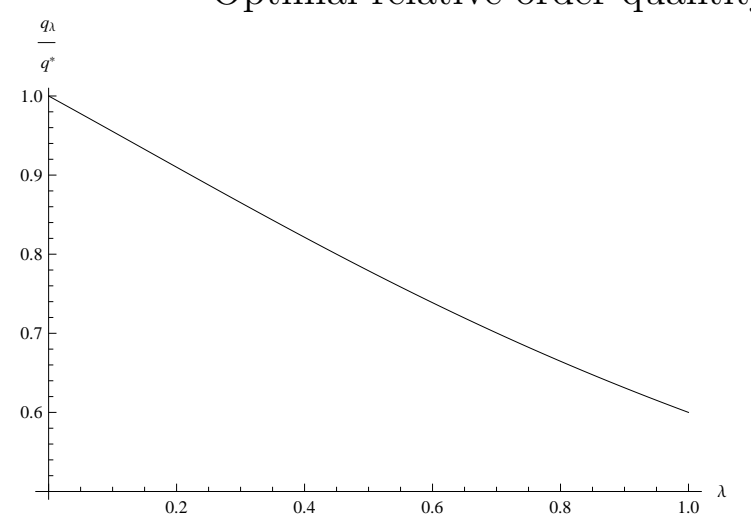

FiguRE 1. $p=1, w=.5$, and $s=.1$.

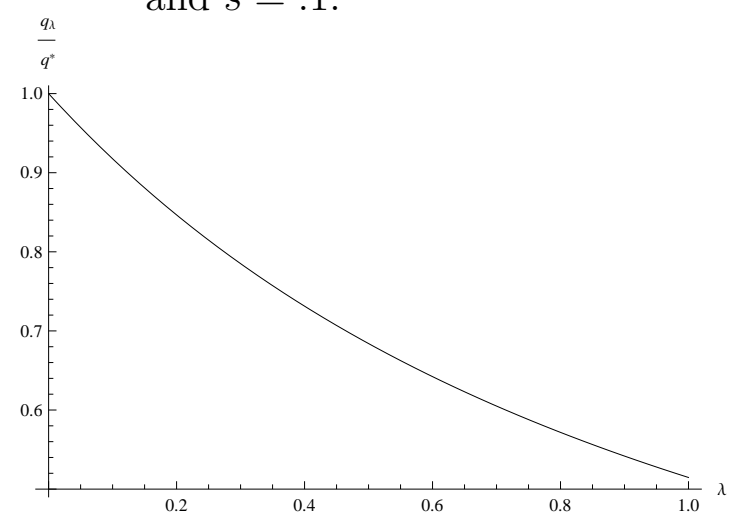

Figure 3. $p=1, w=.9$, and $s=.1$.

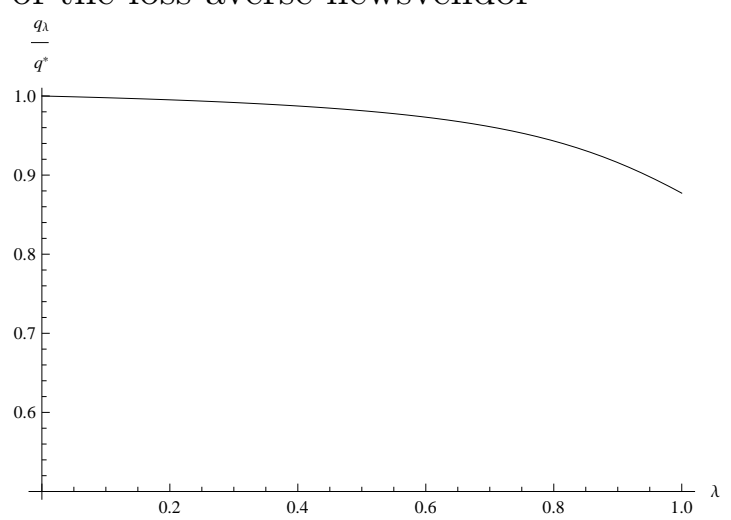

FiguRE 2. $p=1, w=.5$, and $s=.49$.

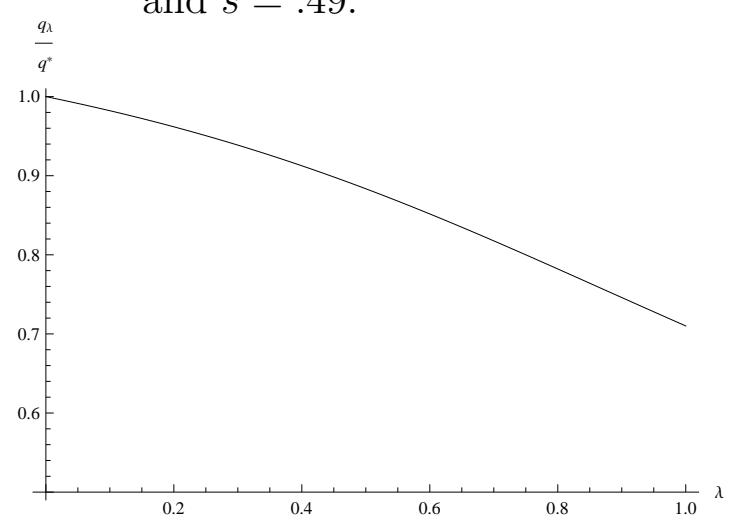

Figure 4. $p=2, w=.5$, and $s=.2$.

of leftovers are low (Figure 2). For low-profit products $\left(F\left(q^{*}\right)<.5\right)$, on the other hand, even a low or moderate degree of loss aversion leads to a considerable reduction in the order quantity (Figure 3). For $\lambda=0.4$ the newsvendor places an order which is less than $75 \%$ of the profit-maximizing quantity.

High degrees of loss aversion. Before proceeding with the analysis of the lossaverse newsvendor's problem for the case with a shortage cost penalty, a few comments regarding Assumption 2 are in order. The assumption $\lambda \leq 1$ guarantees that the newsvendor's expected utility is a strictly concave function in the order quantity. As it becomes apparent from the first derivative (11), even for high degrees of loss aversion, we have $\left.E U^{\prime}(q)\right|_{q=\underline{x}}>0$ and $\left.E U^{\prime}(q)\right|_{q=\bar{x}}<0$. Thus, the optimal order quantity of a highly loss-averse newsvendor $q_{\lambda} \in(\underline{x}, \bar{x})$ and satisfies the firstorder condition (10). Note that all quantities that satisfy the first-order condition (10) are strictly lower than $q^{*}$. Hence, Theorem 2 holds even if Assumption 2 is violated. Moreover, from the second derivative (12) it becomes apparent that the newsvendor's expected utility is strictly concave for all $q$ below a certain threshold and strictly convex for $q$ above this threshold if $\lambda>1$. Thus, we can conclude that $q_{\lambda}$ is uniquely determined by the first-order condition (10) even if Assumption 2 is violated, because the expected utility is strictly decreasing in $q$ when it is convex. 


\section{The Newsvendor Problem with Shortage Cost}

In this section, we assume that if realized demand is higher than the stocked quantity $(x>q)$ then the newsvendor needs to bear a shortage cost penalty $c>0$ on $x-q$ units. Some contributions to the newsvendor literature consider the case of $c \in(w-p, 0)$, which corresponds to situations where $x-q$ units can be ordered and sold during the selling season at a lower margin of $-c$ instead of $p-w \cdot{ }^{10}$ The situation where late orders are feasible is similar to the situation without shortage costs. The newsvendor feels a loss compared to all higher demand states than the actual demand state because profits are increasing in realized demand for a given order quantity. ${ }^{11}$

With "real" shortage costs, however, the profit as a function of realized demand has a unique peak at $x=q$. The profit function is given by (1). The profit is increasing in the demand state for $x<q$ with slope $p-s$. For $x>q$, on the other hand, the profit is decreasing in $x$ with slope $-c$, since stockouts are costly (see Figure 5). For the sake of the argument, suppose that the realized demand is lower than the ordered quantity but not too low. This case is depicted in Figure 5. As before, the newsvendor feels a loss if her actual profit is lower than the profit she could have made for a different realization of demand. Thus, as in the case without a shortage cost, she feels a loss in comparison to all demand states $\tilde{x} \in[x, q]$. Now, however, she feels a loss in comparison only to some demand states $\tilde{x}>q$ but not to all. The upper bound $\phi_{H}$ depends on the realized demand as well as on the ordered quantity. Similarly, for $x>q$ the newsvendor feels a loss compared to all demand states $\tilde{x} \in\left(\phi_{L}, x\right)$. Formally,

$$
\begin{aligned}
\phi_{H}(q, x) & \equiv \max \{z \in[\underline{x}, \bar{x}] \mid \pi(q, z)-\pi(q, x) \geq 0\}, \\
\phi_{L}(q, x) & \equiv \min \{z \in[\underline{x}, \bar{x}] \mid \pi(q, z)-\pi(q, x) \geq 0\} .
\end{aligned}
$$

Notice that $\phi_{L}(q, q)=\phi_{H}(q, q)=q$. Moreover, for an interval of possible demand realizations the threshold values $\phi_{L}$ and $\phi_{H}$ can be equal to the lower and upper bound, respectively, of the demand support. In Figure 5, where $q$ is relatively high, $\phi_{H}(q, x)=\bar{x}$ for all $x \in\left[\underline{x}, \phi_{L}(q, \bar{x})\right]$. The fact that now for each demand realization the newsvendor feels a loss compared to all demand types in an interior interval with bounds depending on $q$ makes the characterization of the optimal order quantity cumbersome. In order to circumvent the problem that the derivative of the threshold values, $\phi_{L}$ and $\phi_{H}$, with respect to the order quantity may not exist, we decompose the newsvendor's problem: First, we characterize the optimal order quantity given the optimal order is relatively high. This case is depicted in Figure 5. Second, we characterize the optimal order given the optimal order is relatively low. Thereafter, we combine these results and show that the newsvendor's problem is globally concave. The first step, in order to do so, is to solve for the critical quantity

\footnotetext{
${ }^{10}$ A notable example is Eeckhouldt, Gollier, and Schlesinger (1995), who analyze a risk-averse and prudent newsvendor.

${ }^{11}$ Suppose the newsvendor can place a late order in case of a stockout at an increased wholesale price $\hat{w}>w$, i.e., $p-\hat{w}=-c>0$. In this case, it can readily be verified that the optimal order quantity, $q_{\lambda}$, is characterized by
}

$$
(\hat{w}-w)-F\left(q_{\lambda}\right)(\hat{w}-s)-\lambda(\hat{w}-s) F\left(q_{\lambda}\right)\left[1-F\left(q_{\lambda}\right)\right]=0,
$$

which implies that $q_{\lambda}<q^{*}$. For $\hat{w}=p(c=0)$ the above first-order condition is equivalent to the first-order condition (10). 


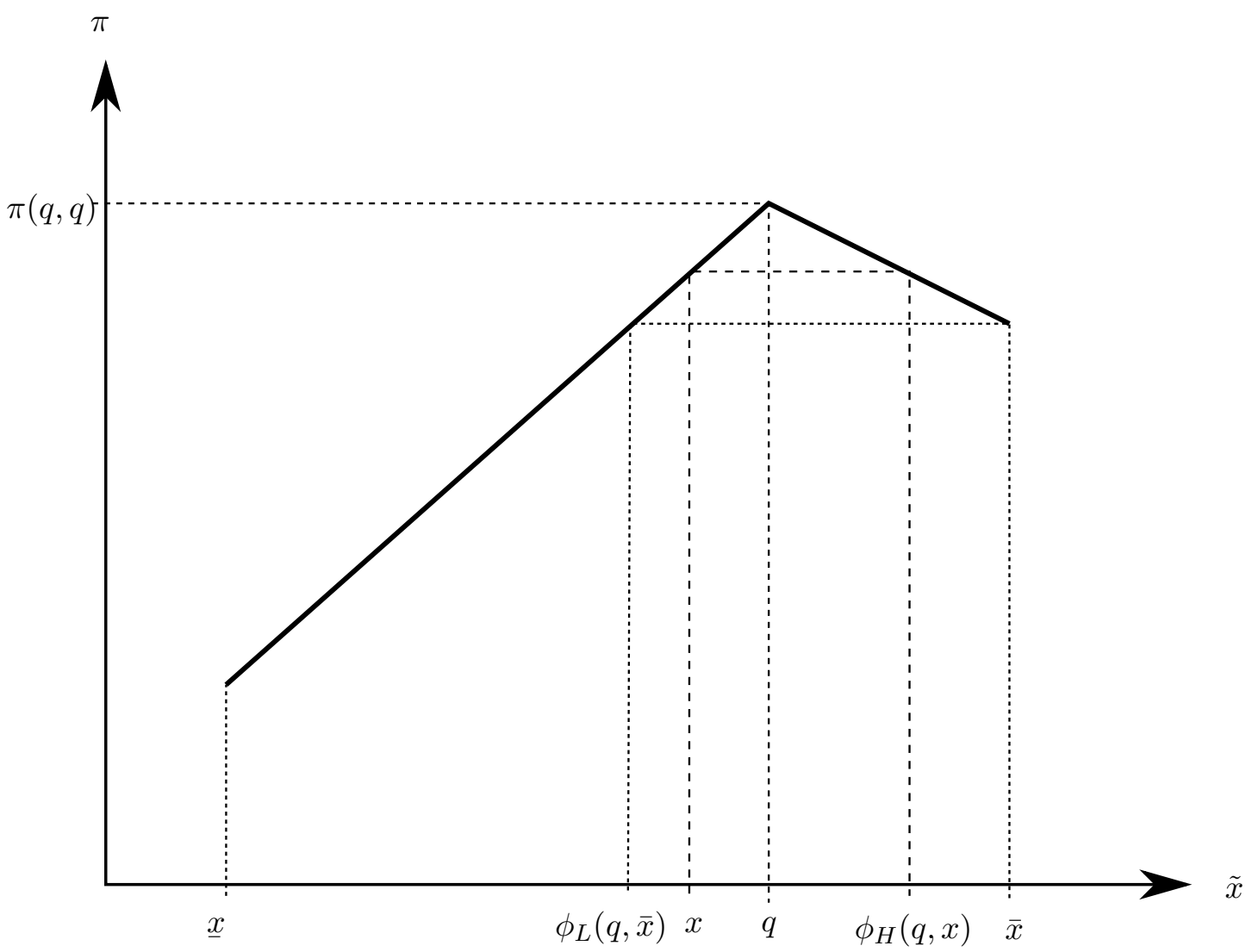

Figure 5. Profit as a function of the possible demand states $\tilde{x}$.

$\bar{q}$ at which $\phi_{L}(q, \bar{x})=\underline{x}$ and $\phi_{H}(q, \underline{x})=\bar{x}$. This critical quantity is given by

$$
\bar{q} \equiv \frac{(p-s) \underline{x}+c \bar{x}}{p-s+c} \in(\underline{x}, \bar{x}) .
$$

Before we proceed with the analysis one remark is in order: Without a shortage penalty the newsvendor feels a loss only in the cases of leftovers. With a positive shortage cost penalty the newsvendor also incurs losses in the cases of stockouts. Put differently, in the case with shortage costs, expectation-based loss aversion leads to endogenous psychological costs for the newsvendor from stockouts as well as from leftovers.

4.1. Relatively High Order Quantity. In this subsection, we presume that $q_{\lambda} \geq$ $\bar{q}$. With $q \geq \bar{q}$ we have $\underline{x}<\phi_{L}(q, x)<q$ for all $x>q$. Moreover, $\phi_{H}(q, x)=\bar{x}$ for $\underline{x} \leq x \leq \phi_{L}(q, \bar{x})$ and $q<\phi_{H}(q, x)<\bar{x}$ for $\phi_{L}(q, \bar{x})<x<q$. Thus, we can decompose the newsvendor's ex-post utility- $\bar{U}$ for $q \geq \bar{q}$-in three parts as follows:

$$
\bar{U}(q, x)= \begin{cases}\bar{u}_{L}(q, x) & \text { if } x \in\left[\underline{x}, \phi_{L}(q, \bar{x})\right), \\ \bar{u}_{M}(q, x) & \text { if } x \in\left[\phi_{L}(q, \bar{x}), q\right), \\ \bar{u}_{H}(q, x) & \text { if } x \in[q, \bar{x}] .\end{cases}
$$

For low realizations of demand the newsvendor feels a loss compared to the profits she would have made in all higher demand states. The reference profits as functions of the hypothetical demand realizations depend on whether the hypothetical demand 
state is higher or lower than the order quantity. Hence,

$$
\begin{aligned}
\bar{u}_{L}(q, x) & =(p-s) x-(w-s) q \\
& -\lambda \int_{x}^{q}(p-s)(z-x) f(z) d z-\lambda \int_{q}^{\bar{x}}[(p-s)(q-x)-c(z-q)] f(z) d z .
\end{aligned}
$$

For intermediate realizations of actual demand $\left(\phi_{L}(q, \bar{x}) \leq x<q\right)$ the newsvendor feels a loss compared to the profits she would have made if realized demand had been between $x$ and $\phi_{H}(q, x)$. Formally,

$$
\begin{aligned}
& \bar{u}_{M}(q, x)=(p-s) x-(w-s) q \\
& -\lambda \int_{x}^{q}(p-s)(z-x) f(z) d z-\lambda \int_{q}^{\phi_{H}(q, x)}[(p-s)(q-x)-c(z-q)] f(z) d z .
\end{aligned}
$$

If the realized demand is relatively high then the newsvendor feels a loss in comparisons to the profits she would have made for lower demand realizations. Precisely, she feels a loss in comparison to all demand states between $\phi_{L}(q, x)$ and $x$,

$$
\begin{aligned}
\bar{u}_{H}(q, x) & =(p+c-w) q-c x \\
& -\lambda \int_{\phi_{L}(q, x)}^{q}\left[(p-s)(z-x)+c(x-q] f(z) d z-\lambda \int_{q}^{x} c(x-z) f(z) d z .\right.
\end{aligned}
$$

For $q \geq \bar{q}$ the newsvendor's expected utility ex ante - using the decomposition (15) can be written as follows

$$
\overline{E U}(q)=\int_{\underline{x}}^{\phi_{L}(q, \bar{x})} \bar{u}_{L}(q, x) f(x) d x+\int_{\phi_{L}(q, \bar{x})}^{q} \bar{u}_{M}(q, x) f(x) d z+\int_{q}^{\bar{x}} \bar{u}_{H}(q, x) f(x) d z .
$$

If a relatively high quantity is optimal then the first-order condition of utility maximization- $E U^{\prime}(q)=0$ - is

$$
\begin{aligned}
& (p+c-w)-(p+c-s) F(q) \\
& +\lambda(p+c-s) F(q)-\lambda(p+c-s)\left[F\left(\phi_{L}(q, \bar{x})\right)\right. \\
& \left.\quad+\int_{\phi_{L}(q, \bar{x})}^{q} F\left(\phi_{H}(q, x)\right) f(x) d x+\int_{q}^{\bar{x}} F\left(\phi_{L}(q, x)\right) f(x) d x\right]=0 .
\end{aligned}
$$

The above first-order condition is hard to interpret. In particular, it is unclear whether the loss-averse newsvendor orders more or less than the profit-maximizing newsvendor. By inspecting (20) we observe, however, that the direction of the bias in order quantity is independent of the degree of loss aversion, only the magnitude of the deviation from the profit-maximizing quantity depends on $\lambda^{12}$ Note that for $q=q^{*}$ the first two terms of (20) cancel each other out. The two remaining terms are both multiplied by $\lambda(p+c-s)$. Thus, whether $\left.\overline{E U^{\prime}}(q)\right|_{q=q^{*}}$ is positive or negative is independent of $\lambda$, because neither $\phi_{L}$ nor $\phi_{H}$ depend on the degree of loss aversion.

\footnotetext{
${ }^{12}$ This statement holds true provided that $E U$ is concave, which is shown below.
} 
4.2. Relatively Low Order Quantity. Now, we presume that $q_{\lambda} \leq \bar{q}$. We use an equivalent approach as in the case $q_{\lambda}>\bar{q}$ and decompose the newsvendor's ex post utility in three parts. For $q \leq \bar{q}$ the expected utility $\underline{E U}(q)$ can be written as

$$
\underline{E U}(q)=\int_{\underline{x}}^{q} \underline{u}_{L}(q, x) f(x) d x+\int_{q}^{\phi_{H}(q, \underline{x})} \underline{u}_{M}(q, x) f(x) d z+\int_{\phi_{H}(q, \underline{x})}^{\bar{x}} \underline{u}_{H}(q, x) f(x) d z .
$$

For $q \leq \bar{q}$ the first-order condition of utility maximization amounts to

$$
\begin{aligned}
& (p+c-w)-(p+c-s) F(q)+\lambda(p+c-s) F(q) \\
& -\lambda(p+c-s)\left[\int_{\underline{x}}^{q} F\left(\phi_{H}(q, x)\right) f(x) d x+\int_{q}^{\phi_{H}(q, \underline{x})} F\left(\phi_{L}(q, x)\right) f(x) d x\right]=0 .
\end{aligned}
$$

Again, the first-order condition is not enlightening regarding the bias in the order quantity of a loss-averse newsvendor. What can be said is that the direction of the bias is independent of $\lambda$, because the sign of $\left.\underline{E U^{\prime}}(q)\right|_{q=q^{*}}$ is independent of $\lambda$. Moreover, the two first-order conditions, (20) and (22), are identical when being evaluated at $q=\bar{q}$.

4.3. Optimal Order Quantity. Having characterized the optimal order of the loss-averse newsvendor separately for the case of high and low order quantities, we are now ready to characterize the overall optimal order quantity $q_{\lambda}$. As a first step it is important to note that $E U(q)$ is a continuous function even at $q=\bar{q}$. Moreover, it can readily be verified that the two first-order conditions, (22) and (20), coincide when being evaluated at $q=\bar{q}$, which implies that $E U(q)$ is differentiable at $q=\bar{q}$. The following result establishes that the newsvendor's problem is concave.

Lemma 1. Given Assumption 2 holds, then the loss-averse newsvendor's expected utility is globally concave and continuously differentiable for all quantities in the range of $[\underline{x}, \bar{x}]$.

Proof. See the Appendix. ${ }^{13}$

Given the newsvendor's expected utility is strictly concave, the next question is whether we can rule out corner solutions. We know that $\underline{x}<\bar{q}<\bar{x}$. It can be shown - using the first-order condition $(22)$ - that $\left.E U^{\prime}(q)\right|_{q=\underline{x}}>0$. Moreover, we have $\left.\overline{E U^{\prime}}(q)\right|_{q=\bar{x}}<0$ by evaluating (20) at $q=\bar{q}$. Thus, the optimal order quantity $q_{\lambda} \in(\underline{x}, \bar{x})$ and thus is implicitly defined by one of the two first-order conditions. Which one of the two first-order conditions characterizes the optimal order quantity depends on the sign of $\left.E U^{\prime}(q)\right|_{q=\bar{q}}$, i.e., whether a higher or lower quantity than $\bar{q}$ is optimal.

Theorem 3. Suppose that Assumption 2 holds. Then, there is a unique optimal order quantity $q_{\lambda}$ characterized by the first-order condition

(i) (22) if $\left.E U^{\prime}(q)\right|_{q=\bar{q}} \leq 0$, and by

(ii) (20) if $\left.E U^{\prime}(q)\right|_{q=\bar{q}}>0$.

What does Theorem 3 tell us about the optimal order quantity of the loss-averse newsvendor? For $\lambda=0$-loss-neutral newsvendor-both first-order conditions are

\footnotetext{
${ }^{13}$ All the proofs that are not given in the main text are relegated to the Appendix A.
} 
identical and coincide with the standard one. As already discussed above, by inspecting the first-order conditions it becomes obvious that the direction of the decision bias does not depend on the degree of loss aversion, i.e., whether the loss-averse newsvendor orders more or less than the profit-maximizing quantity. The extent of the decision bias, however, depends on the degree of loss aversion. ${ }^{14}$

Theorem 4. Suppose that Assumption 2 holds. Then, (i) $d q_{\lambda} / d \lambda>0$ if $q_{\lambda}>q^{*}$, and (ii) $d q_{\lambda} / d \lambda<0$ if $q_{\lambda}<q^{*}$.

In order to gain deeper insights into the behavior of the loss-averse newsvendor, we discuss two examples in the following. The first example, with uniformly distributed demand, shows robustness of our previous findings without a cost of stockouts: the loss-averse newsvendor orders less than the profit-maximizing quantity. The second example, with an exponential demand distribution, shows that the optimal order quantity of the loss-averse newsvendor can exceed the profit-maximizing quantity.

Example 2 (Uniform Distribution). Let $x \sim U[0,1]$ and thus $f(x)=1$ and $F(x)=x$. The critical quantity is $\bar{q}=c /(p+c-s)$. Given this demand distribution a profit-maximizing newsvendor orders the quantity $q^{*}=(p+c-w) /(p+c-s)>\bar{q}$, because $p-w>0$. Hence, the loss-averse newsvendor orders less than the profitmaximizing quantity if and only if $\left.\overline{E U^{\prime}}(q)\right|_{q=q^{*}}<0$. Here, it is immediately obvious that whether $q_{\lambda}$ is greater or smaller than $q^{*}$ does not depend on the degree of loss aversion. For a given $q^{*}>\bar{q}$, we have $\left.\overline{E U^{\prime}}(q)\right|_{q=q^{*}}<0$ if and only if

$$
\begin{aligned}
q^{*}-\frac{p+c-s}{p-s} q^{*}+\frac{c}{p-s}-\int_{\phi_{L}\left(q^{*}, \bar{x}\right)}^{q^{*}} & {\left[\frac{p+c-s}{c} q^{*}+\frac{p-s}{c} x\right] d x } \\
& -\int_{q^{*}}^{1}\left[\frac{p+c-s}{p-s} q^{*}+\frac{c}{p-s} x\right] d x<0 .
\end{aligned}
$$

As we show in the Appendix A, the above inequality is always satisfied for $\bar{q}<q^{*}<$ $1=\bar{x}$.

Proposition 2. Suppose that $x \sim U[0,1]$ and that Assumption 2 holds. Then, the loss-averse newsvendor orders strictly less than the profit-maximizing order quantity, i.e., $q_{\lambda}<q^{*}$.

Example 3 (Exponential Distribution). Suppose that demand $x$ is exponentially distributed with mean $1 / \rho$ and $\rho \in \mathbb{R}_{>0}$. Then $f(x)=\rho e^{-\rho x}$ and $F(x)=$ $1-e^{-\rho x}$. Here, $x=0$ and $\bar{x}=\infty$ which implies that the critical quantity $\bar{q}=\infty$ for $c>0$. With an exponential distribution mass is concentrated on low realizations of $x$. The profit-maximizing quantity is

$$
q^{*}=-\frac{1}{\rho} \ln \left(\frac{w-s}{p+c-s}\right) \in(0, \bar{q}) .
$$

\footnotetext{
${ }^{14}$ Comparative statics of the optimal order quantity $q_{\lambda}$ with respect to price and cost parameters are undetermined. It is intricate to determine the sign of say $d q_{\lambda} / d p$ because $\phi_{L}(\cdot)$ and $\phi_{H}(\cdot)$ do not only depend on $p$ indirectly via $q_{\lambda}$ but also directly.
} 
The optimal order of the loss-averse newsvendor $q_{\lambda}$ is characterized by the first-order condition (22):

$$
\begin{array}{r}
\frac{p+c-w}{p+c-s}-1+e^{-\rho q_{\lambda}}+\lambda\left(1-e^{-\rho q_{\lambda}}\right)-\lambda \int_{0}^{q_{\lambda}}\left(1-e^{-\rho\left(\frac{p+c-s}{c} q_{\lambda}-\frac{p-s}{c} x\right)}\right) \rho e^{-\rho x} d x \\
-\lambda \int_{q_{\lambda}}^{\frac{p+c-s}{c}}\left(1-e^{-\rho\left(\frac{p+c-s}{p-s} q_{\lambda}-\frac{c}{p-s} x\right)}\right) \rho e^{-\rho x} d x=0 .
\end{array}
$$

The first-order condition (24) is intricate to solve analytically. Numerical solutions of the optimal order quantity, $q_{\lambda}$, of the loss-averse newsvendor as function of the shortage cost $c$ and the wholesale price $w$ are plotted in Figure 6 and Figure 7, respectively. The dotted line depicts the order placed by the loss-averse newsvendor $q_{\lambda}$ and the solid line is the profit-maximizing order $q^{*}$. For the numerical simulation we assumed that $\lambda=1$ and $\rho=2$ which implies that $\mathbb{E}[x]=1 / 2$ as in Example 2 . The presumed price structure is as follows: $p=1000$, and $s=250$. In Figure 6 we set $w=990$ and vary $c$ from 0 to 200 . In Figure 7 we set $c=120$ and vary the wholesale price $w \in[975,999]$.

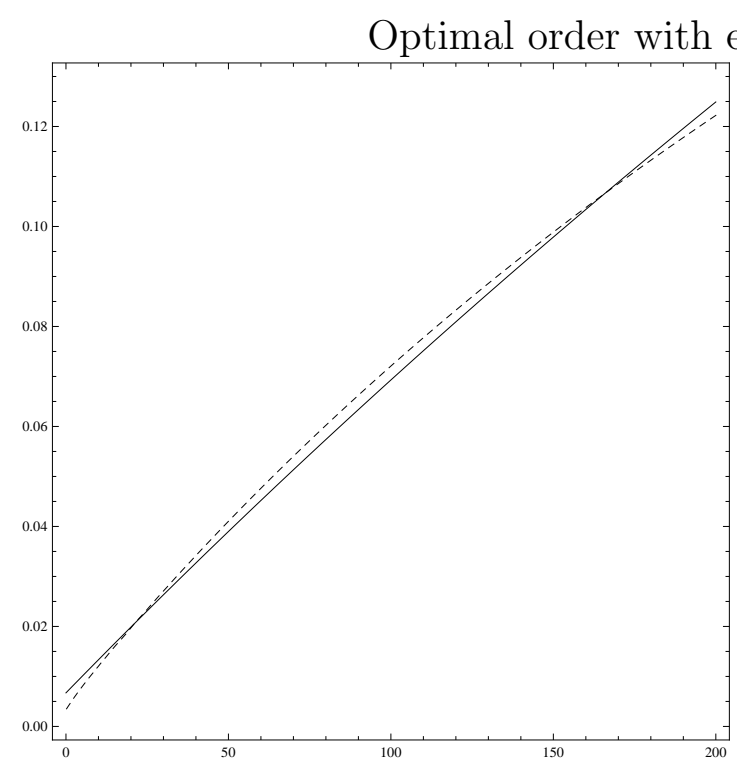

Figure 6. $q_{\lambda}(c)$ for $c \in[0,200]$.

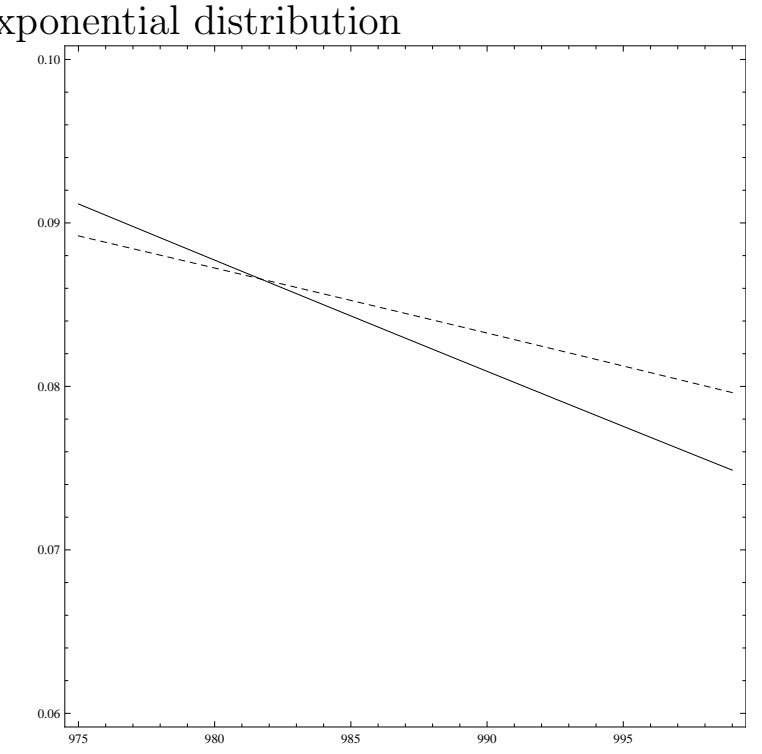

Figure 7. $q_{\lambda}(w)$ for $w \in[975,999]$.

The numerical simulation reveals that the optimal order placed by the loss-averse newsvendor can exceed the profit-maximizing quantity. If there is a penalty associated with stockouts, then the expectation-based loss-averse newsvendor suffers from a psychological pain not only in the case of leftovers but also in the case of stockouts. The loss-averse newsvendor chooses a quantity ex ante in order to balance the trade-off between maximizing profits and minimizing the expected loss. This is the reason why the loss-averse newsvendor sometimes places higher orders than the profit-maximizing newsvendor if there is a unit shortage cost penalty incurred on the excess demand. In this case, there is no psychological disutility only if realized demand exactly meets the order quantity, i.e., $q=x$. While here this is an implication following from the assumption of an expectation-based loss-averse newsvendor, 
Ho, Lim, and Cui (2010) directly impose this assumption in their model of a biased newsvendor.

Observation 1. If there are positive costs associated with stockouts, then the order placed by the loss-averse newsvendor can be higher as well as lower than the profitmaximizing quantity.

\section{Regret Averse Newsvendor}

The economic as well as the psychological literature discusses several types of reference-dependent preferences. Two important notions of reference-dependent preferences are loss aversion and regret aversion. So far we analyzed a model of expectation-based loss aversion. Here, the newsvendor is disappointed about the draw by nature. The newsvendor moans that realized demand is not closer to her actual order quantity and this causes her psychological pain. ${ }^{15}$ A regret-averse newsvendor, on the other hand, is not disappointed about the move by nature but regrets the choice she has made ex ante (Bell, 1982; Loomes and Sugden, 1982). Put differently, a regret-averse newsvendor moans about the fact that she has not chosen an order quantity closer to the realized demand. Schweitzer and Cachon (2000) and Ho, Lim, and Cui (2010) analyze a regret-averse newsvendor. Both papers posit that the newsvendor's psychological disutility is proportional to the ex-post error in order quantity $|q-x|$ but does not depend on the loss in profits associated with these suboptimal orders. The regret-averse newsvendor exhibits in both papers the so called "pull-to-center" bias, a behavior often found in experimental tests of the newsvendor model. ${ }^{16}$ According to the pull-to center bias actual orders are distorted towards the median of the demand distribution compared to the optimal order.

In the following, we will analyze the decision of a regret-averse newsvendor, whose psychological cost is related to the loss in profits due to the suboptimal decision ex ante. More precisely, the newsvendor's ex-post utility is given by

$$
U(q, x)=\pi(q, x)-\lambda\left[\max _{z}\{\pi(z, x)\}-\pi(q, x)\right]
$$

with $\lambda \geq 0$. As Schweitzer and Cachon (2000) and Ho, Lim, and Cui (2010), we posit that the newsvendor regrets that she has not placed the ex-post optimal order. Note that $\max _{z} \pi(z, x)=\pi(x, x)$. Hence, for low realizations of demand, $x<q$, the newsvendor's ex-post utility is given by

$$
\hat{U}(q, x)=(p-s) x-(w-s) q-\lambda(w-s)(q-x) .
$$

For high realizations of demand, $x \geq q$, the ex-post utility is

$$
\tilde{U}(q, x)=(p+c-w) q-c x-\lambda(p+c-w)(x-q) .
$$

Our model is equivalent to Ho, Lim, and Cui (2010)'s if one sets the psychological cost of overages $d_{o}=\lambda(w-s)$ and the psychological cost of underages $d_{u}=\lambda(p+$ $c-w)$. So the crucial distinction between our model and Ho, Lim, and Cui (2010)'s is that the relative psychological aversion of leftovers to stockouts is free in Ho, Lim,

\footnotetext{
${ }^{15}$ The feature of expectation-based loss aversion according to Köszegi and Rabin $(2006,2007)$ that the decision maker is disappointed about the unlucky move made by nature is shared by models of disappointment aversion (Bell, 1985; Loomes and Sugden, 1986; Gul, 1991).

${ }^{16}$ See for instance Schweitzer and Cachon (2000). Recently, Lau, Bearden, and Hasija (2012) argue that the pull-to-center effect might be an artifact of aggregate data, which does not exist for individual order decisions.
} 
and Cui (2010), whereas it is determined by the price structure in our model. As the next proposition shows, this minor change of making the psychological disutility proportional to the actual loss in profits has a fundamental effect on the result.

Proposition 3. Suppose the newsvendor is regret averse. Then, the newsvendor's expected utility is strictly concave and maximized at the profit-maximizing order quantity $q^{*}$.

If the psychological disutility is proportional to the actual loss in profits, then regret aversion does not lead to a bias in order quantities. ${ }^{17}$

\section{Conclusion}

This paper analyzes the order placed by an expectation-based loss-averse newsvendor. The loss-averse newsvendor is disappointed if the actual demand does not coincide with her ordered quantity. More precisely, the newsvendor incurs a psychological cost - a loss - in the case of stockouts as well as leftovers. These psychological costs arise endogenously due to the applied loss-aversion concept à la Köszegi and Rabin (2006). With her ordering decision, the newsvendor balances the ex-ante expected losses due to stockouts against the expected losses due to leftovers. If the monetary shortage penalty is low, then the newsvendor is more concerned about not having sold all ordered units and in consequence places an order ex ante which is too low from the profit-maximizing point of view. If, on the other hand, the shortage penalty is high the loss-averse newsvendor might order a quantity which is higher than the quantity stocked by a profit-maximizing newsvendor.

We believe that the analysis of an expectation-based loss-averse newsvendor helps us to understand the order decisions made by procurement managers as well as experimental subjects. Loss aversion is a well-established departure from standard preferences, in particular from EUT. Even though loss aversion is a prevalent behavioral bias which describes the behavior of many decision makers not all people are loss averse (Choi, Fisman, Gale, and Kariv, 2007). In the light of the recent evidence provided by Lau, Bearden, and Hasija (2012) that the distribution of orders in newsvendor experiments is typically bimodal, we are tempted to conjecture that one modal point might be described by our theory of a loss-averse newsvendor. There are several other biases, errors in judgment, and heuristic thinking that might play a role in newsvendor settings and might therefore interact with loss aversion. A more thorough empirical analysis of individual order decisions seems to be required in order to shed more light on these issues.

\section{Appendix A}

Proof of Lemma 1. The first derivatives of expected utility for the two cases, $q \leq \bar{q}$ and $q>\bar{q}$, are provided in the proof of Theorem 3 below. Note that the derivatives of the threshold demand states, $\phi_{H}$ and $\phi_{L}$, with respect to $q$ are strictly positivegiven the derivative exists, see (A.3) and (A.4).

\footnotetext{
${ }^{17}$ This finding is not surprising in the light of Maier and Rüger (2009), who show that regret aversion, in contrast to loss aversion, has no effect on even-order risk preferences such as risk aversion.
} 
Taking the second-derivative of expected utility with respect to $q$ for $q \leq \bar{q}$ yields (first derivative is given by (A.14))

(A.1) $E U^{\prime \prime}(q)=-(p+c-s) f(q)(1-\lambda)$

$-\lambda(p+c-s)\left[\int_{\underline{x}}^{q} f\left(\phi_{H}(q, x)\right) f(x) \frac{d \phi_{H}}{d q} d x+\int_{q}^{\phi_{H}(q, \underline{x})} f\left(\phi_{L}(q, x)\right) f(x) \frac{d \phi_{L}}{d q} d x\right]<0$,

because $\lambda \leq 1$ by Assumption 2 .

The second derivative of expected utility for the case $q>\bar{q}$ is given by (differentiating (A.19))

$(\mathrm{A} .2) \quad \overline{E U^{\prime \prime}}(q)=-(p+c-s) f(q)(1-\lambda)$

$-\lambda(p+c-s)\left[\int_{\phi_{L}(q, \bar{x})}^{q} f\left(\phi_{H}(q, x)\right) f(x) \frac{d \phi_{H}}{d q} d x+\int_{q}^{\bar{x}} f\left(\phi_{L}(q, x)\right) f(x) \frac{d \phi_{L}}{d q} d x\right]<0$,

by Assumption 2 .

Noting that not only $\left.\underline{E U}(q)\right|_{q=\bar{q}}=\left.\overline{E U}(q)\right|_{q=\bar{q}}$ but also $\left.\underline{E U^{\prime}}(q)\right|_{q \nearrow_{\bar{q}}}=\left.\overline{E U^{\prime}}(q)\right|_{q \searrow \bar{q}}$, which implies that $E U(q)$ is continuously differentiable, completes the proof.

Proof of Theorem 3. As a first step, note that the loss thresholds, $\phi_{L}$ and $\phi_{H}$ can be stated as follows

$$
\begin{aligned}
\phi_{L}(q, x) & =\max \left\{\underline{x}, \frac{p+c-s}{p-s} q-\frac{c}{p-s} x\right\}, \\
\phi_{H}(q, x) & =\min \left\{\bar{x}, \frac{p+c-s}{c} q-\frac{p-s}{c} x\right\},
\end{aligned}
$$

and thus both are (weakly) increasing in $q$. The proof is split in two parts. First, we analyze the case $q \leq \bar{q}$ and thereafter $q>\bar{q}$.

Step $1(q \leq \bar{q})$ : The ex-post utility can be decomposed into three parts - depending on the realization of demand $x$-as follows

$$
\underline{U}(q, x)= \begin{cases}\underline{u}_{L}(q, x) & \text { if } x \in[\underline{x}, q), \\ \underline{u}_{M}(q, x) & \text { if } x \in\left[q, \phi_{H}(q, \underline{x})\right), \\ \underline{u}_{H}(q, x) & \text { if } x \in\left[\phi_{H}(q, \underline{x}), \bar{x}\right] .\end{cases}
$$

For low realizations of demand the ex-post utility is

$$
\begin{aligned}
\underline{u}_{L}(q, x) & =(p-s) x-(w-s) q-\lambda(p-s) \int_{x}^{q}(z-x) f(z) d z \\
& \left.+\lambda c \int_{q}^{\phi_{H}(q, x)}(z-q)\right] f(z) d z-\lambda(p-s)(q-x)\left[F\left(\phi_{H}(q, x)\right)-F(q)\right] .
\end{aligned}
$$


By taking the derivative of $\underline{u}_{L}$ with respect to $q$, we obtain

$$
\begin{aligned}
\frac{\partial \underline{u}_{L}(q, x)}{\partial q} & =-(w-s)+\lambda c\left[\phi_{H}(q, x)-q\right] f\left(\phi_{H}(q, x)\right) \frac{d \phi_{H}}{d q} \\
-\lambda c \int_{q}^{\phi_{H}(q, x)} f(z) d z-\lambda(p-s)\left[F\left(\phi_{H}(q, x)\right)-F(q)\right] & \\
& -\lambda(p-s)(q-x)\left[f\left(\phi_{H}(q, x)\right) \frac{d \phi_{H}}{d q}-f(q)\right] .
\end{aligned}
$$

Using the fact that $(p-s)(q-x)=c\left(\phi_{H}-q\right)$, we can simplify the above equation to

$$
\frac{\partial \underline{u}_{L}(q, x)}{\partial q}=-(w-s)+\lambda(p+c-s)\left[F\left(\phi_{H}(q, x)\right)-F(q)\right] .
$$
by

For intermediate realizations of demand, the newsvendor's ex-post utility is given

$$
\begin{aligned}
\underline{u}_{M}(q, x)=(p+c-w) q-c x-\lambda c(x-q)\left[F(q)-F\left(\phi_{L}(q, x)\right)\right] & \\
& +\lambda(p-s) \int_{\phi_{L}(q, x)}^{q}(q-z) f(z) d z-\lambda c \int_{q}^{x}(x-z) f(z) d z .
\end{aligned}
$$

The partial derivative with respect to $q$ is

$$
\frac{\partial \underline{u}_{M}(q, x)}{\partial q}=(p+c-w)+\lambda(p+c-s)\left[F(q)-F\left(\phi_{L}(q, x)\right)\right] .
$$

In order to obtain the derivative (A.10), we used the fact that $(p-s)\left(q-\phi_{L}\right)-$ $c(x-q)=0$ for intermediate realizations of $x$.

Finally, for high realizations of $x$ the ex-post utility is

$$
\begin{aligned}
\underline{u}_{H}(q, x)=(p+c-w) & q-c x-\lambda c(x-q) F(q) \\
& +\lambda(p-s) \int_{\underline{x}}^{q}(q-z) f(z) d z-\lambda c \int_{q}^{x}(x-z) f(z) d z .
\end{aligned}
$$

Taking the partial derivative of (A.11) with respect to $q$ yields

$$
\frac{\partial \underline{u}_{H}(q, x)}{\partial q}=(p+c-w)+\lambda(p+c-s) F(q) .
$$

For $q \leq \bar{q}$, the newsvendor's expected utility is

$$
\underline{E U}(q)=\int_{\underline{x}}^{q} \underline{u}_{L}(q, x) f(x) d x+\int_{q}^{\phi_{H}(q, \underline{x})} \underline{u}_{M}(q, x) f(x) d z+\int_{\phi_{H}(q, \underline{x})}^{\bar{x}} \underline{u}_{H}(q, x) f(x) d z .
$$

The partial derivative of expected utility with respect to the order quantity is

$$
\begin{aligned}
& \underline{E} \underline{U}^{\prime}(q)=\underline{u}_{L}(q, q) f(q)+\int_{\underline{x}}^{q} \frac{\partial}{\partial q} \underline{u}_{L}(q, x) f(x) d x \\
& +\underline{u}_{M}\left(q, \phi_{H}(q, \underline{x})\right) f\left(\phi_{H}(q, \underline{x})\right) \frac{d \phi_{H}}{d q}-\underline{u}_{M}(q, q) f(q)+\int_{q}^{\phi_{H}(q, \underline{x})} \frac{\partial}{\partial q} \underline{u}_{M}(q, x) f(x) d x \\
& -\underline{u}_{H}\left(q, \phi_{H}(q, \underline{x})\right) f\left(\phi_{H}(q, \underline{x})\right) \frac{d \phi_{H}}{d q}+\int_{\phi_{H}(q, \underline{x})}^{\bar{x}} \frac{\partial}{\partial q} \underline{u}_{H}(q, x) f(x) d x .
\end{aligned}
$$


After using the specific expressions for the ex-post utility levels, $\underline{u}_{L}, \underline{u}_{M}, \underline{u}_{H}$, and their derivatives and noting that $\phi_{L}\left(q, \phi_{H}(q, \underline{x})\right)=\underline{x}$ and thus $\underline{u}_{M}\left(q, \phi_{H}(q, \underline{x})\right)=$ $\underline{u}_{H}\left(q, \phi_{H}(q, \underline{x})\right)$, we can simplify the above formula to

$$
\begin{aligned}
& E U^{\prime}(q)=(p+c-w)-(p+c-s) F(q)+\lambda(p+c-s) F(q) \\
& \quad-\lambda(p+c-s)\left[\int_{\underline{x}}^{q} F\left(\phi_{H}(q, x)\right) f(x) d x+\int_{q}^{\phi_{H}(q, \underline{x})} F\left(\phi_{L}(q, x)\right) f(x) d x\right] .
\end{aligned}
$$

To conclude the first step of the proof note that $\left.\underline{E U^{\prime}}(q)\right|_{q=\underline{x}}=(p+c-s)>0$.

Step $2(q>\bar{q})$ : The decomposition of ex-post utility in the three parts is given in the main text (15) as well as the expressions of these three parts. Taking the derivative of (16) with respect to $q$ yields

$$
\frac{\partial \bar{u}_{L}(q, x)}{\partial q}=-(w-s)-\lambda(p+c-s)[1-F(q)]
$$

Taking the derivative of (17) with respect to $q$ and noting that - for the considered $x$ values - $(p-s)(q-x)=c\left(\phi_{H}-q\right)$, leads to

$$
\frac{\partial \bar{u}_{M}(q, x)}{\partial q}=-(w-s)-\lambda(p+c-s)\left[F\left(\phi_{H}(q, x)-F(q)\right] .\right.
$$

Finally, the derivative of (18) with respect to $q$ is given by,

$$
\frac{\partial \bar{u}_{H}(q, x)}{\partial q}=(p+c-w)+\lambda(p+c-s)\left[F(q)-F\left(\phi_{L}(q, x)\right] .\right.
$$

In order to obtain the above expression, we used that $(p-s)\left(q-\phi_{L}\right)=c(x-q)$ for high realizations of $x$

Thus, the first-order condition of utility maximization - provided that $q>\bar{q}$ - is given by $\overline{E U^{\prime}}(q)=0$, with

$$
\begin{aligned}
& \overline{E U^{\prime}}(q)=\int_{\underline{x}}^{\phi_{L}(q, \bar{x})} \frac{\partial}{\partial q} \bar{u}_{L}(q, x) f(x) d x+u_{L}\left(q, \phi_{L}(q, \bar{x})\right) f\left(\phi_{L}(q, \bar{x})\right. \\
& +\int_{\phi_{L}(q, \bar{x})}^{q} \frac{\partial}{\partial q} \bar{u}_{M}(q, x) f(x) d x+u_{M}(q, q) f(q)-u_{M}\left(q, \phi_{L}(q, \bar{x})\right) f\left(\phi_{L}(q, \bar{x})\right. \\
& +\int_{q}^{\bar{x}} \frac{\partial}{\partial q} \bar{u}_{H}(q, x) f(x) d x-u_{H}(q, q) f(q) .
\end{aligned}
$$

Note that $\phi_{H}\left(q, \phi_{L}(q, \bar{x})\right)=\bar{x}$ and thus $\bar{u}_{L}\left(q, \phi_{L}(q, \bar{x})\right)=\bar{u}_{M}\left(q, \phi_{L}(q, \bar{x})\right)$. The derivative of expected utility can be simplified to

$$
\begin{aligned}
E U^{\prime}(q)= & (p+c-w)-(p+c-s) F(q) \\
& +\lambda(p+c-s) F(q)-\lambda(p+c-s)\left[F\left(\phi_{L}(q, \bar{x})\right)\right. \\
& \left.\quad+\int_{\phi_{L}(q, \bar{x})}^{q} F\left(\phi_{H}(q, x)\right) f(x) d x+\int_{q}^{\bar{x}} F\left(\phi_{L}(q, x)\right) f(x) d x\right] .
\end{aligned}
$$

Noting that $\left.E U^{\prime}(q)\right|_{q=\bar{x}}=-(w-s)<0$ and using the fact that $E U(q)$ is strictly concave and continuously differentiable by Lemma 1 completes the proof. 
Proof of Theorem 4. First, we consider the case $q_{\lambda} \leq \bar{q}$, in which $q_{\lambda}$ is implicitly characterized by (22). Let

$$
\underline{\Psi}\left(q_{\lambda}\right) \equiv F\left(q_{\lambda}\right)-\left[\int_{\underline{x}}^{q_{\lambda}} F\left(\phi_{H}\left(q_{\lambda}, x\right)\right) f(x) d x+\int_{q_{\lambda}}^{\phi_{H}\left(q_{\lambda}, \underline{x}\right)} F\left(\phi_{L}\left(q_{\lambda}, x\right)\right) f(x) d x\right] .
$$

Note that $\underline{\Psi}\left(q_{\lambda}\right)>0$ if $q_{\lambda}>q^{*}$ and $\underline{\Psi}\left(q_{\lambda}\right)<0$ if $q_{\lambda}<q^{*}$. By the implicit function theorem, from (22), we get:

$$
\begin{aligned}
\frac{d q_{\lambda}}{d \lambda}\left[(1-\lambda) f\left(q_{\lambda}\right)+\lambda \int_{\underline{x}}^{q_{\lambda}}\right. & f\left(\phi_{H}\left(q_{\lambda}, x\right)\right) f(x) \frac{d \phi_{H}}{d q} d x \\
& \left.+\lambda \int_{q_{\lambda}}^{\phi_{H}\left(q_{\lambda}, \underline{x}\right)} f\left(\phi_{L}\left(q_{\lambda}, x\right)\right) f(x) \frac{d \phi_{L}}{d q} d x\right]=\underline{\Psi}\left(q_{\lambda}\right) .
\end{aligned}
$$

The desired result follows immediately from the fact that the term in square brackets on the left-hand side is positive by Assumption 2 .

Now, we consider the case $q_{\lambda}>\bar{q}$. The optimal order is characterized by (20). We define

$$
\begin{aligned}
\bar{\Psi}\left(q_{\lambda}\right) \equiv F\left(q_{\lambda}\right) & -\left[F\left(\phi_{L}\left(q_{\lambda}, \bar{x}\right)\right)\right. \\
& \left.+\int_{\phi_{L}\left(q_{\lambda}, \bar{x}\right)}^{q_{\lambda}} F\left(\phi_{H}\left(q_{\lambda}, x\right)\right) f(x) d x+\int_{q_{\lambda}}^{\bar{x}} F\left(\phi_{L}\left(q_{\lambda}, x\right)\right) f(x) d x\right] .
\end{aligned}
$$

By the implicit function theorem, from (20), we get:

$$
\begin{aligned}
\frac{d q_{\lambda}}{d \lambda}\left[(1-\lambda) f\left(q_{\lambda}\right)+\lambda \int_{\phi_{L}\left(q_{\lambda}, \bar{x}\right)}^{q_{\lambda}}\right. & f\left(\phi_{H}\left(q_{\lambda}, x\right)\right) f(x) \frac{d \phi_{H}}{d q} d x \\
& \left.\quad+\lambda \int_{q_{\lambda}}^{\bar{x}} f\left(\phi_{L}\left(q_{\lambda}, x\right)\right) f(x) \frac{d \phi_{L}}{d q} d x\right]=\bar{\Psi}\left(q_{\lambda}\right) .
\end{aligned}
$$

Again, the term in squared brackets on the left-hand side of (A.23) is positive by Assumption 2 and thus $d q_{\lambda} / d \lambda>0$ if and only if $\bar{\Psi}\left(q_{\lambda}\right)>0$.

Proof of Proposition 2. The loss-averse newsvendor orders less than $q^{*}$ if

$$
\begin{aligned}
-\frac{c}{p-s} q^{*}+\frac{c}{p-s}-\int_{\frac{p+c-s}{p-s} q^{*}-\frac{c}{p-s}}^{q^{*}} & {\left[\frac{p+c-s}{c} q^{*}+\frac{p-s}{c} x\right] d x } \\
& -\int_{q^{*}}^{1}\left[\frac{p+c-s}{p-s} q^{*}+\frac{c}{p-s} x\right] d x<0 .
\end{aligned}
$$

Let $\alpha:=c /(p-s)$. With this definition the inequality (A.24) simplifies to

$$
\alpha-\alpha q^{*}-\int_{(1+\alpha) q^{*}-\alpha}^{q^{*}}\left[\frac{1+\alpha}{\alpha} q^{*}+\frac{1}{\alpha} x\right] d x-\int_{q^{*}}^{1}\left[(1+\alpha) q^{*}-\alpha x\right] d x<0 .
$$

Rearranging the above inequality yields

$$
\left(q^{*}\right)^{2}-\frac{1+2 \alpha}{1+\alpha} q^{*}+\frac{\alpha}{1+\alpha}<0 .
$$


The roots of inequality (A.26) are

$$
\hat{q}_{1}=\frac{\alpha}{1+\alpha}=\frac{c}{p+c-s}=\bar{q}>q^{*}, \quad \text { and } \quad \hat{q}_{2}=1=\bar{x}>q^{*} .
$$

The inequality (A.26) is satisfied for all quantities $q^{*} \in\left(\hat{q}_{1}, \hat{q}_{2}\right)$, which concludes the proof.

Proof of Proposition 3. The regret-averse newsvendor's expected utility is

$$
\begin{aligned}
E U(q) & =(1+\lambda) \int_{\underline{x}}^{\bar{x}} \pi(q, x) f(x) d x-\lambda \int_{\underline{x}}^{\bar{x}} \pi(x, x) f(x) d x \\
& =(1+\lambda) E \pi(q)-\text { constant. }
\end{aligned}
$$

Thus, the regret-averse newsvendor faces the same problem as the profit-maximizing newsvendor up to an affine transformation.

\section{REFERENCES}

Abeler, J., A. Falk, L. Goette, and D. Huffman (2011): "Reference Points and Effort Provision," The American Economic Review, 101(2), 470-492.

Arrow, K. J., T. Harris, and J. MarschaK (1951): "Optimal Inventory Policy," Econometrica, 19(3), 250-272.

Becker-Peth, M., E. Katok, and U. W. Thoenemann (2011): "Designing Contracts for Irrational but Predictable Newsvendors," Working Paper, University of Cologne - Germany.

BELl, D. E. (1982): "Regret in Decision Making under Uncertainty," Operations Research, 30(5), pp. 961-981.

(1985): "Disappointment in Decision Making under Uncertainty," Operations Research, 33(1), pp. 1-27.

Bostian, A. A., C. A. Holt, and A. M. Smith (2008): "Newsvendor "Pull-toCenter" Effect: Adaptive Learning in a Laboratory Experiment," Manufacturing \& Service Operations Management, 10(4), 590-608.

Choi, S., R. Fisman, D. Gale, and S. Kariv (2007): "Consistency and Heterogeneity of Individual Behavior under Uncertainty," American Economic Review, 97(5), 1921-1938.

Croson, D. C., R. Croson, and Y. Ren (2008): "How to Manage an Overconfident Newsvendor," Working Paper, University of Texas-Dallas.

Edgeworth, F. Y. (1888): "The Mathematical Theory of Banking," Journal of the Royal Statistical Society, 51(1), 113-127.

Eeckhouldt, L., C. Gollier, and H. Schlesinger (1995): "The risk-averse (and prudent) newsboy," Management Science, 41(5), 786-794.

ERicson, K. M. M., AND A. Fuster (2011): "Expectations as Endowments: Evidence on Reference-Dependent Preferences from Exchange and Valuation Experiments," The Quarterly Journal of Economics, 126(4), 1879-1907.

Fisher, M., AND A. Raman (1996): "Reducing the Cost of Demand Uncertainty through Accurate Response to Early Sales," Operations Research, 44(1), pp. 8799.

Gul, F. (1991): "A Theory of Disappointment Aversion," Econometrica, 59(3), pp. $667-686$. 
Herweg, F., And K. MierendorfF (forthcoming): "Uncertain Demand, Consumer Loss Aversion, and Flat-Rate Tariffs," Journal of the European Economic Association.

Herweg, F., D. Müller, And P. Weinschenk (2010): "Binary Payment Schemes: Moral Hazard and Loss Aversion," American Economic Review, 100(5), 2451-2477.

Ho, T. H., N. Lim, And T. H. Cui (2010): "Reference Dependence in Multilocation Newsvendor Models: A Structural Analysis," Management Science, 56(11), 1891-1910.

Kahneman, D., And A. TVersky (1979): "Prospect Theory: An Analysis of Decision under Risk," Econometrica, 47(2), pp. 263-292.

Katok, E., A. Lathrop, W. Tarantino, and S. H. Xu (2001): "Jeppesen Uses a Dynamic-Programming-Based DSS to Manage Inventory," Interfaces, 31(6), pp. 54-65.

Köszegi, B., And M. RABin (2006): "A Model of Reference-Dependent Preferences," Quarterly Journal of Economics, 121(4), 1133-1165.

- (2007): "Reference-Dependent Risk Attitudes," American Economic Review, 97(4), 1047-1073.

Lau, N., J. N. Bearden, and S. HasiJa (2012): "Distributional Features of Newsvendor Behavior," INSEAD Working Paper.

Loomes, G., And R. Sugden (1982): "Regret Theory: An Alternative Theory of Rational Choice Under Uncertainty," The Economic Journal, 92(368), pp. 805824.

(1986): "Disappointment and Dynamic Consistency in Choice under Uncertainty," The Review of Economic Studies, 53(2), 271-282.

MacCrimmon, K. R., And D. Wehrung (1988): Taking Risks: The Management of Uncertainty. Free Press, New York.

MAiER, J., AND M. RÜGER (2009): "Reference-Dependent Risk Preferences of Higher Orders," Working Paper, University of Munich.

Moutaz, And Khouja (1999): "The single-period (news-vendor) problem: literature review and suggestions for future research," Omega, 27(5), $537-553$.

Qin, Y., R. Wang, A. J. Vakharia, Y. Chen, and M. M. Seref (2011): "The newsvendor problem: Review and directions for future research," European Journal of Operational Research, 213(2), 361 - 374.

Schweitzer, M. E., and G. P. Cachon (2000): "Decision Bias in the Newsvendor Problem with a Known Demand Distribution: Experimental Evidence," Management Science, 46(3), 404-420.

Su, X. (2008): "Bounded Rationality in Newsvendor Models," Manufacturing \&6 Service Operations Management, 10(4), 566-589.

Wang, C. X., And S. Webster (2009): "The loss-averse newsvendor problem," Omega, 37(1), 93-105.

Wang, C. X., S. Webster, and N. C. Suresh (2009): "Would a risk-averse newsvendor order less at a higher selling price?," European Journal of Operational Research, 196(2), 544-553.

Ludwig-Maximilian-University Munich and CESifo

E-mail address: fabian.herweg@lmu.de 